\title{
Comparative Analysis of Antioxidative Activity of Flavonoids Using HPLC-ED and Photometric Assays
}

\author{
Pawel Piszcz • Magdalena Woźniak • \\ Monika Asztemborska • Bronisław K. Glód
}

Received: 9 August 2013 / Accepted: 25 November 2013 / Published online: 11 December 2013

(C) The Author(s) 2013. This article is published with open access at Springerlink.com

\begin{abstract}
The present investigation reports on the application of a new antioxidant activity assay for the examination of flavonoids. It has been shown that the high-performance liquid chromatography with electrochemical detection (HPLC-ED) measurements allow to obtain additional information about the antioxidative properties of pure compounds by measuring their half-wave potential, the chromatographic peak height, and the product of the peak height and exponent of potential. In comparison to the classical electrochemical measurements, the HPLC-ED is characterized by a much smaller detection limit. The results were compared with the standard photometric measurement based on 1,1-diphenyl-2-picrylhydrazyl radicals. The possible antioxidant activity forecasting is also discussed.
\end{abstract}

Keywords Antioxidants $\cdot$ DPPH $\cdot$ Flavonoids $\cdot$ Free radicals $\cdot$ HPLC-ED

\section{Introduction}

Flavonoids are the main bioactive components of vegetables and fruits. They have antioxidant, antimicrobial, antiallergenic, anti-inflammatory, insecticidal, and light-screening activities and act as photoreceptors, visual attractors, and feeding repellants. Therefore, they are predominant in the human diet, and intake may reach up to $1 \mathrm{~g}$ /day (Pietta 2000). Their

P. Piszcz $\cdot$ M. Woźniak $\cdot$ B. K. Głód $(\bowtie)$

Department of Analytical Chemistry, Institute of Chemistry, Faculty

of Science, University of Natural Sciences and Humanities, 3 Maja

54, 08-110 Siedlce, Poland

e-mail: bkg@onet.eu

M. Asztemborska

Institute of Physical Chemistry, Polish Academy of Sciences,

Kasprzaka 44/52, 01-224 Warsaw, Poland antioxidant activity is regarded as scavenging free radicals by donation of electrons.

Many methods of antioxidant activity evaluation have been developed (Wantusiak et al. 2011; Głód et al. 2012), such as scavenging activity determination using 1,1-diphenyl-2picrylhydrazyl (DPPH) or Folin-Ciocalteu (FCR) reagent. Photometric (Uma Devi et al. 2008) and chromatographic (Głód et al. 2000, 2012) assays are, generally, based on competition between the sample and the sensor for reaction with the free radicals. Often, flavonoids show inconsistent antioxidant activities, depending on the assay used (Zhang et al. 2011).

A good measure of the antioxidant activity of pure compounds, like flavonoids, is their oxidation potential. It can be determined potentiometrically (Brainina et al. 2007) or using the voltammetric measurements (Alonso et al. 2003). These measurements cannot be applied to real samples with complex biological (food, blood, etc.) matrix, containing many various antioxidants at different concentrations. Recently, we have proposed an original assay of the total antioxidant potential (TAP) measurement, based on the reverse-phase highperformance liquid chromatography (RP-HPLC) antioxidant separation followed by electrochemical (amperometric) detection (Wantusiak et al. 2012). This assay was used to the TAP estimation of various food samples. In the anodic potential range, only oxidation current is observed. It means that chromatographic peaks originate from the antioxidants. The TAP measure is the total surface area of all chromatographic peaks. It is dependent on the potential of the working electrode.

The main purpose of this work was to study the antioxidative properties of 15 flavonoids (Fig. 1) by using high-performance liquid chromatography with electrochemical detection (HPLCED) (involving measurement of chromatographic peak height); moreover, the structural parameters (energy of the highest occupied molecular orbital (HOMO) orbital) were calculated. The results (obtained using photometric assay) were compared to the antioxidant activity related to the DPPH radicals. 


\section{Materials and Methods}

Instrumentation

Chromatographic measurements were performed by means of a chromatograph comprising an Interface Box, four-channel Smartline Manager 5000 with Degasser K-5004, Solvent Organizer K-1500, Dynamic Mixing Chamber, HPLC Pump Smartline 1000, ClinLab Digital Amperometric Detector EC3000 (Recipe, Munich, Germany) with a glassy carbon working electrode (reference electrode- $\mathrm{Ag} / \mathrm{AgCl}$, auxiliary electrode-Pt), Smartline Diode Array Detector 2800, Autosampler Smartline 3900 (all from Knauer GmbH, Berlin, Germany), and Smartline 4000 Column Thermostat (Industrial Electronics, Langenzersdorf, Austria). Samples were separated on a Cosmosil RP-C18-MS-II column (5 $\mu \mathrm{m}, 250 \times$ $4.6 \mathrm{~mm}$ I.D.; Nacalai, USA). The system was controlled, and data acquisition was performed on an IBM PC-type computer with ClarityChrom V 2.6 2007 software.

Photometric measurements were performed on a Helios Epsilon spectrophotometer (Thermo Scientific, USA).

The application HyperChem ${ }^{\mathrm{TM}}$ Release 8.0.7 (molecular modeling system; Hypercube, Gainesville, FL, USA) for Windows was used for the structural parameter (energy of the HOMO orbital) evaluation.

pH was measured using an OP-208/1 pH meter (Radelkis, Budapest, Hungary) with an OSH 10-10 electrode (MetronWCF, Czekanów, Poland).

\section{Reagents}

Gallic acid, sodium perchlorate, DPPH, and HPLC-grade methanol were obtained from Sigma (St. Louis, MO, USA). All other reagents (Fluka, Buchs, Switzerland; Alchem, Inform, and POCh, Poland) were of analytical reagent grade and were used without further purification. Water was distilled three times from quartz. Mobile phases were filtered through a 0.22- $\mu \mathrm{m}$ membrane filter (Millipore, Bedford, USA).

Various flavonoid standards including (+)-catechin, (-)-epicatechin, hesperetin, hesperidin, neohesperidin, naringin, naringenin, flavanone, 7,8-benzoflavanone, 6methoxyflavanone, 6-hydroxyflavanone, 4'-hydroxyflavanone, 2 '-hydroxyflavanone, quercetin, and rutin were purchased from Sigma-Aldrich, USA (Fig. 1).

Chromatographic/Amperometric Measurements of Antioxidant Activity

Chromatographic experiments were performed at a flow rate of $1.0 \mathrm{~mL} \mathrm{~min}^{-1}$. The column was stabilized at $20( \pm 0.1)^{\circ} \mathrm{C}$ by passage of mobile phase for $0.5 \mathrm{~h}$ prior to the chromatographic measurements. Methanol + sodium perchlorate $(80+20 \%$, $v / v)$ in water $\left(0.1 \mathrm{~mol} \mathrm{~L}^{-1}\right)$ was used as a mobile phase; 1.0 - mmol L ${ }^{-1}$ stock solutions of the analyzed flavonoids were prepared in methanol and diluted to $0.1 \mathrm{mmol} \mathrm{L}^{-1}$ before use. Using an autosampler, 20- $\mu \mathrm{L}$ samples were injected. Output signal from the electrochemical detector was continuously displayed on the computer (Wantusiak et al. 2012).

\section{Photometric DPPH Assay}

The antioxidative activity of the flavonoid samples was assessed on the basis of the scavenging activity of the stable DPPH free radical according to previous reports (Brand-Williams et al. 1995; Sharma and Bhat 2009; Huang et al. 2012) with slight modifications. To $700 \mu \mathrm{L}$ of $0.1 \mathrm{mmol} \mathrm{L}^{-1} \mathrm{DPPH}$ in methanol, $1.4 \mathrm{~mL}$ of a $0.01-\mathrm{mmol} \mathrm{L}^{-1}$ flavonoid solution in methanol was added. The mixture was shaken vigorously and left to stand for $20 \mathrm{~min}$ at room temperature in the dark. The change of absorbance at $517( \pm 0.2) \mathrm{nm}$ was measured. The antioxidant activity was expressed as percentage of DPPH radical concentration $\left(\mathrm{AA}=\left[\left(A_{\text {blank }}-A_{\text {sample }}\right) / A_{\text {blank }}\right] \times\right.$ $100[\%])$, where $A_{\text {blank }}$ is the absorbance of the DPPH radical solution and $A_{\text {sample }}$ is the absorbance of the DPPH radical solution after the addition of $10 \mu \mathrm{mol} \mathrm{L}{ }^{-1}$ of sample solution.

\section{Results and Discussion}

TAP can be measured using RP-HPLC separation of antioxidants followed by their amperometric measurements (Wantusiak et al. 2012). At anodic potentials, only antioxidants are recorded on chromatograms. In this paper, it will be discussed whether this assay can be used to the antioxidant activity (AA) estimations of pure compounds. Although in this case AA can be simply expressed as a red/ox potential, the disadvantage of these methods is very small sensitivity. In voltammetry, current is measured as a function of potential. However, much higher signals (than those in traditional voltammetric measurements) are observed in the flowing (among them HPLC) systems. This is due to (1) neglected capacity current (measurements are performed at a constant potential) and (2) huge and stable convectional current (current is directly proportional to the flow rate of depolarizer to the electrode). A very small volume of the electrochemical detector cell means that the mobile phase flows through it at a very high linear flow rate. This, in turn, causes a large convection current and, consequently, lowers detection limit and yields higher sensitivity of chromatographic measurements with electrochemical detection than that of voltammetric measurements. So, 0.1-mmol L ${ }^{-1}$ flavonoid solutions are undetectable using voltammetry, hence the advantage of hydrodynamically recorded voltammograms. Changes of convection current are responsible for the current (chromatographic signal) changes observed at the dead column volume (Fig. 2). The current flowing through the system depends also on the 
Fig. 1 Structures of the investigated flavonoids

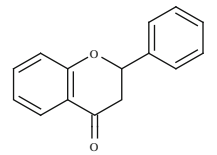

flavanone<smiles>COc1ccc2c(c1)C(=O)CC(c1ccccc1)O2</smiles>

6-methoxyflavanone<smiles>O=C1CC(c2ccc(Cl)cc2)Oc2cc(O)cc(O)c21</smiles>

naringenin<smiles>Cc1ccc([C@@H]2Oc3cc(O)cc(O)c3C[C@H]2O)cc1O</smiles>

(+)catechin

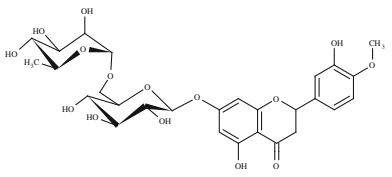

hesperidin

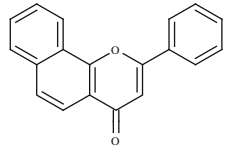

7,8-benzoflavanone<smiles>O=C1CC(c2ccccc2O)Oc2ccccc21</smiles>

2'-hydroxyflavanone<smiles>COc1ccc([C@H]2CC(=O)c3c(O)cc(O)cc3O2)cc1O</smiles>

hesperetin<smiles>O=c1c(O)c(-c2ccc(O)c(O)c2)oc2cc(O)cc(O)c12</smiles>

quercetin

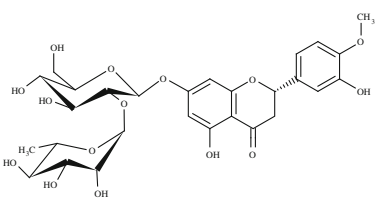

neohesperidin<smiles>O=C1CC(c2ccccc2)Oc2ccc(O)cc21</smiles>

6-hydroxyflavanone<smiles>O=C1CC(c2ccc(O)cc2)Oc2ccccc21</smiles>

4'-hydroxyflavanone<smiles>Cc1ccc([C@@H]2Oc3cc(O)cc(O)c3C[C@H]2C)cc1O</smiles>

(-)epicatechin

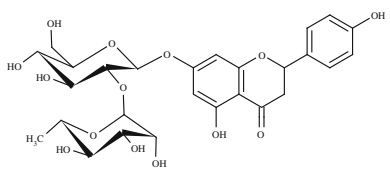

naringin

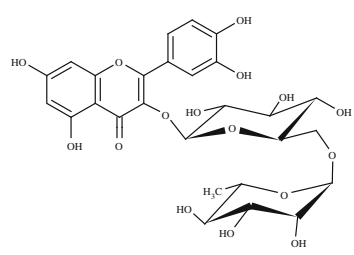

rutin changes of the solvent dielectric constant and on the concentration of the supporting electrolyte. Usually, polar solvents and strong electrolytes are used as mobile phase in RP-HPLC. They are eluted near the dead column volume. Hence, when a sample is not dissolved in the mobile phase, then, often, the additional peak is observed in the dead column volume. Therefore, the described measurements cannot be obtained using flow injection systems, without a column. The column is necessary to separate the solute peak from the dead volume. The reported method is based on isocratic elution, and for the given mobile phase conditions, the solutes were eluted at $2.8 \pm$ $0.1 \mathrm{~min}$, while the dead time was approximately $2 \mathrm{~min}$ (Fig. 2).

The hydrodynamic voltammograms of five different flavonoids are presented in Fig. 3. It is easy to see their characteristic wave shapes. They differ significantly in comparison to the classical voltammograms. These waves are more similar to waves obtained polarographically, using a rotating disc electrode or microelectrode. It turned out (Table 1) that among the investigated flavonoids, quercetin is the strongest antioxidant, which is consistent with the available literature data (RiceEvans et al. 1996). Quercetin is characterized by the smallest half-wave potential (i.e., potential at which the wave current is equal to one half of its maximum current) and the highest chromatographic peaks, particularly the peak obtained at $0.1 \mathrm{~V}$. At higher potentials, the additional waves are observed, similarly to the voltammetric results (Brett and Ghica 2003). Quercetin is also the best electron donor from all investigated flavonoids. It contains a catechol moiety, namely, the $3^{\prime}, 4^{\prime}$ dihydroxyl electron-donating group in ring $\mathrm{B}$. The oxidation mechanism proceeds in sequential steps, related to the catechol moiety and 3-hydroxyl group. The oxidation of the 


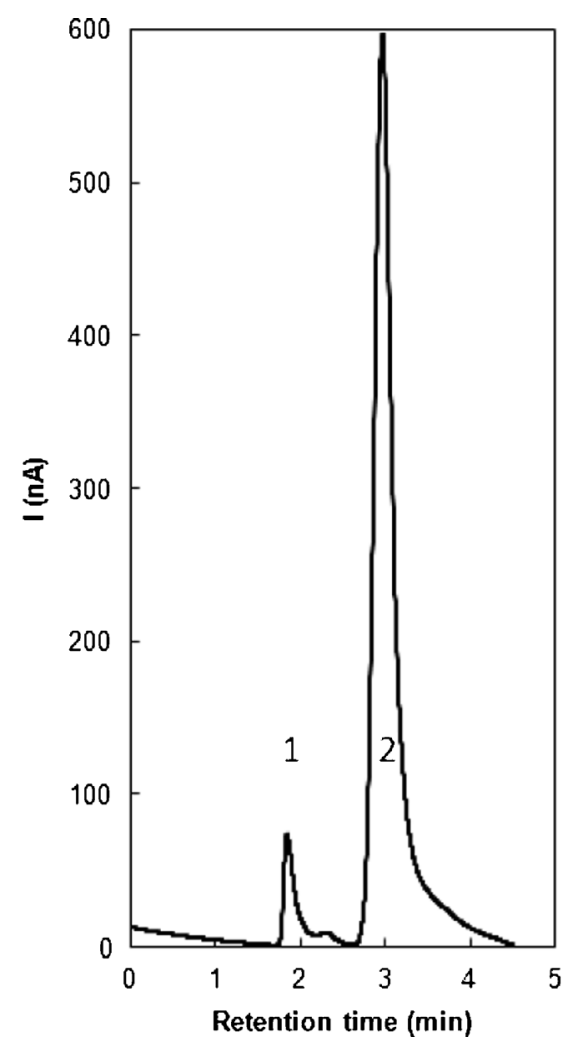

Fig. 2 RP-HPLC-ED chromatogram of 6-hydroxyflavanone (2), separated from the dead column volume (1). Chromatographic conditions: column - Cosmosil RP-C18-MS-II (Nacalai; $5 \mu \mathrm{m}, 250 \times 4.6 \mathrm{~mm}$ ); mobile phase $-0.1 \mathrm{~mol} \mathrm{~L}^{-1} \mathrm{NaClO}_{4} / \mathrm{MeOH}$ 80:20\%(v/v); flow rate$1.0 \mathrm{~mL} \mathrm{~min}^{-1}$, electrochemical detector with the glassy carbon used as a working electrode; potential $-0.9 \mathrm{~V}$ vs. $\mathrm{Ag} / \mathrm{AgCl}$

catechol electron-donating group occurs first at a low positive potential and involves a two-electron-two-proton reversible reaction and forms $o$-quinone. The following steps are oxidation of the 3-hydroxyl group located in ring $\mathrm{C}$ and the resorcinol group (5,7-dihydroxyl) in ring A undergoing an irreversible reaction (Pierożyński and Zielińska 2011). Quercetin is also characterized by the highest antioxidant activity measured in relation to the DPPH radical and the smallest energy of the HOMO orbital (Table 1).

Catechin $\left(E_{1 / 2}=0.55 \mathrm{~V}\right)$ is structurally similar to quercetin; it has also a catechol group in ring $\mathrm{B}$, resorcinol moiety in ring $\mathrm{A}$, and hydroxyl group at position 3 in ring C. However, the lack of a carbonyl group as well as double bond in ring $\mathrm{C}$ causes the catechin to be unconjugated and characterized by less mesomeric structures, and therefore, it is a weaker antioxidant (Janeiro and Brett 2004). Even weaker antioxidative properties are observed for those compounds in which one hydroxyl group is changed to a methoxyl one, as it is observed for hesperitin $\left(E_{1 / 2}=0.73 \mathrm{~V}\right)$, or removed (4'hydroxyflavanone, $\left.E_{1 / 2}=0.55 \mathrm{~V}\right)$. Also, glycosylation increases the half-wave potential (hesperidin, $E_{1 / 2}=0.55 \mathrm{~V}$ ). Glycosylation additionally decreases the height of the chromatographic peak because of a decrease of the diffusion coefficient. Finally, 6-methoxyflavanone, 7,8benzoflavanone, and flavanone, where the B ring is unsubstituted phenyl, are not oxidized electrochemically (Table 1).

A good correlation between peak heights and $E_{1 / 2}$ was found (Table 1), but only for peaks recorded at the smallest potentials. Due to the nonlinearity of hydrodynamic voltammograms (for example, see Fig. 3), such correlation is not observed for peak heights recorded at higher potentials. Comparison of the peak heights obtained at the various potentials gives deeper insight into the antioxidative sample properties. According to the Randles-Sevcik equation, the oxidation
Fig. 3 Hydrodynamic voltammograms of quercetin (circle), (+)-catechin (square), 4'hydroxyflavanone (diamond), hesperedin (triangle), and flavanone (asterisk). Chromatographic conditions are as in Fig. 2 except the working electrode range of potentials$0.0-1.2 \mathrm{~V}$ vs. $\mathrm{Ag} / \mathrm{AgCl}$. The points represent the results from three independent measurements. In all cases, RSD $\leq 5 \%$

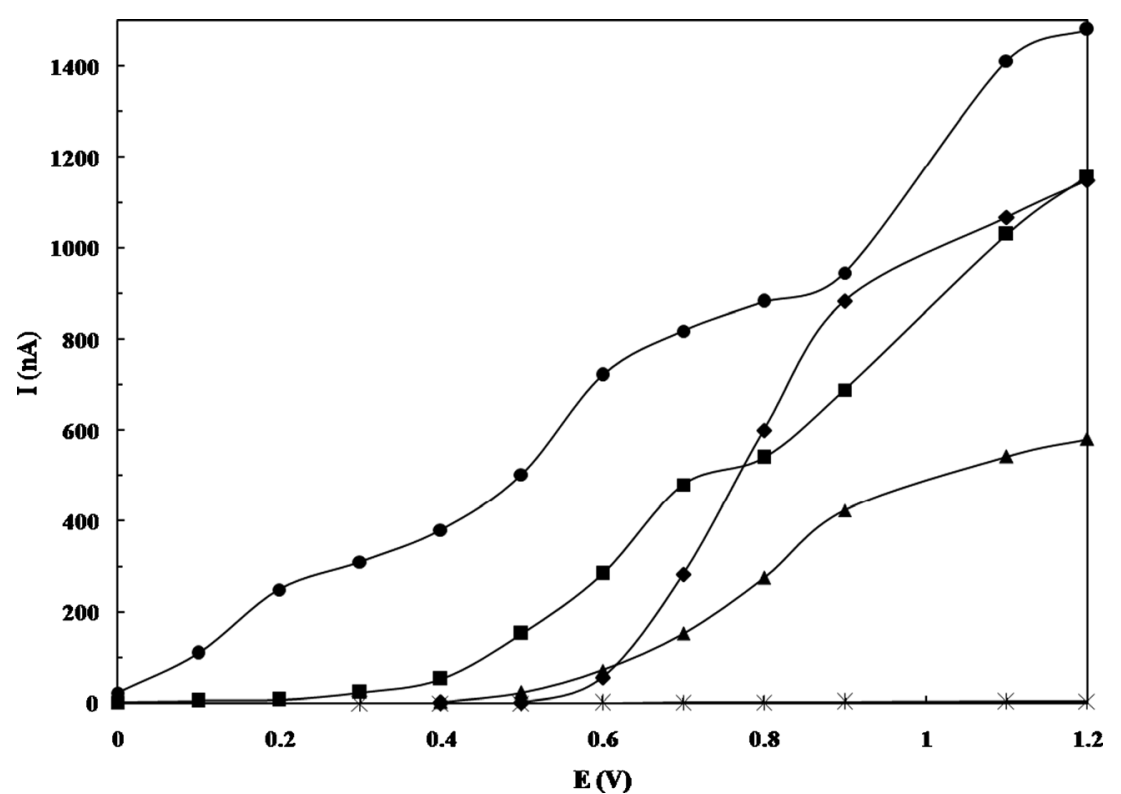


Table 1 Antioxidant activity of flavonoids

\begin{tabular}{|c|c|c|c|c|c|c|c|}
\hline Sample & $E_{1 / 2}(\mathrm{~V})$ & $\mathrm{AA}^{\mathrm{DPPH}}(\%)$ & $e^{(2.08-E)} h^{0.4}(\mathrm{nA})$ & $E^{\mathrm{HOMO}}(\mathrm{eV})$ & $h^{0.4}(\mathrm{nA})^{\mathrm{a}}$ & $h^{0.7}(\mathrm{nA})^{\mathrm{a}}$ & $h^{0.9}(\mathrm{nA})^{\mathrm{a}}$ \\
\hline Quercetin & 0.12 & 75.3 & 28.98 & -8.55 & 381.1 & 811.4 & 930.6 \\
\hline$(-)$-Epicatechin & 0.35 & 52.8 & 24.25 & -8.67 & 263.8 & 864.6 & $1,060.7$ \\
\hline Rutin & 0.52 & 65.2 & 11.2 & -8.86 & 121.6 & 322.4 & 356.6 \\
\hline$(+)$-Catechin & 0.55 & 43.9 & 14.11 & -8.85 & 60.8 & 508.6 & 688.8 \\
\hline Hesperetin & 0.73 & 10.6 & 11.51 & -8.81 & 3.0 & 336.1 & 764.1 \\
\hline 6-Hydroxyflavanone & 0.73 & 1.3 & 8.06 & -8.96 & 0.9 & 218.7 & 593.5 \\
\hline 4'-Hydroxyflavanone & 0.74 & 0.7 & 12.04 & -9.17 & 1.4 & 285.7 & 884.1 \\
\hline 2'-Hydroxyflavanone & 0.75 & 0.8 & 11.02 & -9.15 & 19.4 & 256.6 & 760.0 \\
\hline Neohesperidin & 0.76 & 3.0 & 11.07 & -8.87 & 3.7 & 287.8 & 825.6 \\
\hline Hesperidin & 0.80 & 9.5 & 6.27 & -8.82 & 4.3 & 336.1 & 423.5 \\
\hline Naringin & 0.82 & 1.5 & 7.43 & -9.25 & 3.8 & 91.3 & 555.4 \\
\hline Naringenin & 0.83 & 0.9 & 5.71 & -9.14 & 0.9 & 25.3 & 416.7 \\
\hline 6-Methoxyflavanone & - & 0.5 & 0 & -8.87 & 0.3 & 1.3 & 2.9 \\
\hline 7,8-Benzoflavanone & - & 0.4 & 0 & -8.69 & 0.1 & 1.2 & 2.0 \\
\hline Flavanone & - & 0.4 & 0 & -9.36 & 0.3 & 1.99 & 3.6 \\
\hline
\end{tabular}

$E_{1 / 2}$ (volts) is the half-wave potential of the first oxidation step; $\mathrm{AA}^{\mathrm{DPPH}}$ (percent) is the antioxidant activity related to DPPH; $E^{\mathrm{HOMO}}$ (volts) is the energy of the highest occupied molecular orbital; $h^{0.4,0.7,0.9}$ (nanoamperes) are the heights of the chromatographic peaks obtained at various potentials of the working electrode; and $e^{(2.08-E)} h^{0.4}$ (nanoamperes) is the product of the chromatographic peak height obtained at $0.4 \mathrm{~V}$ by exponent from the potential related to the reduction potential of hydroxyl radicals

${ }^{\mathrm{a}} \mathrm{RSD}<4 \%$, for all experiments

current is directly proportional to the solute concentration and to the square root of the depolarizer diffusion coefficient. Hence, smaller peaks are observed for glycosides (as an example, compare hesperitin and hesperedin or quercetin and rutin).

From the above discussion, it turned out that the antioxidative properties of flavonoids can be expressed as half-wave potential and or height of the chromatographic peak. The former value describes the sample antioxidative activity. The latter depends on the sample concentration and, indirectly, on its antioxidative properties. The aim of this paper was to find a more universal assay of AA measurements. It should enable the AA measurement of single compounds as well as any biological or food sample. Therefore, it is impossible to derive proper equation because it is impossible to take any assumption, like that the reaction is reversible or that it is in equilibrium. It is expected that AA will be proportional to the electrode potential at which the chromatographic measurements of the peak height are performed. Therefore, we propose to measure the potential in relation to the redox potential of
Fig. 4 Correlation between (circle) $E^{\mathrm{OH}}-E_{1 / 2}$ and $\mathrm{TAP}^{\mathrm{HPLC}-}$

$\mathrm{ED}$ as well as between (square) $h^{0.4}$ and TAP ${ }^{\mathrm{HPLC}-\mathrm{ED}}$

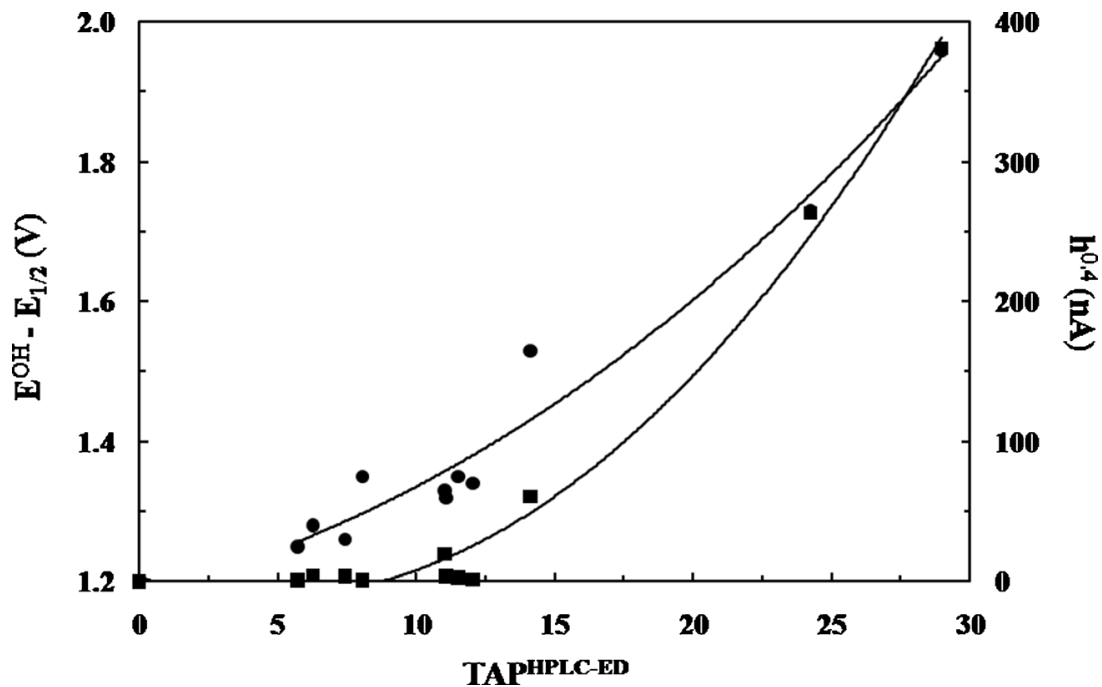


hydroxyl radical $\left(E^{\mathrm{OH}}-E\right)$. This new potential value is directly proportional to the antioxidant activity. In the next step, the product of peak height and potential should be used as an AA measure. The strong antioxidants are oxidized at low potential and low reaction current (the chromatographic peak height). Therefore, we purpose to use the exponential function. Finally, a new assay of the total antioxidant potential (named $\mathrm{TAP}^{\mathrm{HPLC}-\mathrm{ED}}$ ) can be performed using the equation which is expressed as follows:

$$
\mathrm{TAP}^{\mathrm{HPLC}-\mathrm{ED}}=\sum_{i=1}^{n} \mathrm{AA}_{i}=\sum_{i=1}^{n} e^{\left(E^{\mathrm{OH}}-E\right)} \cdot h_{i}^{E}
$$

It turned out (Table 1) that TAP values are directly correlated with the $E_{1 / 2}$ and $h^{0.4}$ values, as it is presented in Fig. 4. In this case, only qualitative correlation is expected. TAP measured using the proposed equation is less sensitive to the experimental inaccuracies.

In the literature, many methods of the antioxidative measurements are described (Głód et al. 2000). One of the most popular is assay based on the photometric measurements of DPPH radicals (Brand-Williams et al. 1995; Huang et al. 2012). The main mechanism of the reaction between flavonoid and DPPH is the formation of the phenoxyl radical, characterized by many resonance structures. Also, in this case, quercetin is characterized by the strongest antioxidant activity (Table 1), and flavanone does not react with DPPH. Generally, a good correlation is obtained between $\mathrm{TAP}^{\mathrm{HPLC}-\mathrm{ED}}$ and AA measured using DPPH and peak heights recorded at $0.4 \mathrm{~V}$. Because DPPH is a weak radical and oxidant, therefore, $\mathrm{AA}^{\mathrm{DPPH}}$ is correlated with the electrochemical oxidation at relatively low potentials.

The antioxidant activity of flavonoids has been the subject of several studies in the past years, and important structure-activity relationships of the antioxidant activity have been established. It was interesting to check if there are any correlations between AA and HOMO (highest occupied orbital) energies. The HOMO orbitals and map of the electrostatic potential were calculated using semiempirical AM1 method (HyperChem ${ }^{\mathrm{TM}}$ Release 8.0.7) using optimization conditions described in the literature (Lien et al. 1999; Seyoum et al. 2006). The results are presented in Table 1. When the energy difference between HOMO and LUMO orbitals is small, then the electron can be easily triggered. In the case when this difference increases, the greater energy is needed to excite an electron. In summary, the lower the HOMO energy, the slower is the reaction observed. Flavonoids are characterized by similar HOMO energy values because of their structural similarity.

\section{Conclusions}

1. HPLC-ED assay can be used for the estimations of the antioxidant activity of pure compounds as it was shown for flavonoids.

2. As an antioxidant activity measure, the first half-wave potential and/or height of the chromatographic peak can be used.

3. The new TAP measure is proposed: $\mathrm{TAP}^{\mathrm{HPLC}-\mathrm{ED}}=\sum_{i=1}^{n} \mathrm{AA}_{i}=\sum_{i=1}^{n} e^{\left(E^{\mathrm{OH}}-E\right)} \cdot h_{i}^{E}$.

4. The proposed assay is much more sensitive than the electrochemical measurements because of the lack of capacity current and very big convection current.

5. The results were confirmed with those obtained using DPPH assay as well as energy of HOMO orbitals.

Conflict of Interest Paweł Piszcz declares that he has no conflict of interest. Magdalena Woźniak declares that she has no conflict of interest. Monika Asztemborska declares that she has no conflict of interest. Bronisław Głód declares that he has no conflict of interest. This article does not contain any studies with human or animal subjects.

Open Access This article is distributed under the terms of the Creative Commons Attribution License which permits any use, distribution, and reproduction in any medium, provided the original author(s) and the source are credited.

\section{References}

Alonso AM, Guillén DA, Barroso CG (2003) Development of an electrochemical method for the determination of antioxidant activity. Application to grape-derived products. Eur Food Res Technol 216:445

Brainina KZ, Ivanova AV, Sharafutdinova EN, Lozovskaya EL, Shkarina EI (2007) Potentiometry as a method of antioxidant activity investigation. Talanta 71:13

Brand-Williams W, Cuvelier ME, Berset C (1995) Use of a free radical method to evaluate antioxidant activity. LWT Food Sci Technol 28:25

Brett AMO, Ghica ME (2003) Electrochemical oxidation of quercetin. Electroanalysis 15:1745

Głód BK, Czapski GA, Haddad PR (2000) Application of highperformance liquid chromatography to the investigation of free radical reactions in biological systems. TrAC - Trends Anal Chem 19:492

Głód BK, Piszcz P, Czajka J, Zarzycki PK (2012) Evaluation of total antioxidant potential of selected biogenic polyamines, non-alcoholic drinks and alcoholic beverages using improved RP-HPLC assay involving fluorescence detection. Food Chem 131:1026

Huang WY, Zhang HC, Liu WX, Li CY (2012) Survey of antioxidant capacity and phenolic composition of blueberry, blackberry, and strawberry in Nanjing. J Zhejiang Univ Sci B 13:94 
Janeiro P, Brett AMO (2004) Catechin electrochemical oxidation mechanisms. Anal Chim Acta 518:109

Lien EJ, Ren S, Bui HH, Wang R (1999) Quantitative structure-activity relationship analysis of phenolic antioxidants. Free Radic Biol Med $26: 285$

Pierożyński B, Zielińska D (2011) Electrosorption of quercetin on glassy carbon electrode. J Electroanal Chem 651:100

Pietta PG (2000) Flavonoids as antioxidants. J Nat Prod 63:1035

Rice-Evans CA, Miller NJ, Paganga G (1996) Structure-antioxidant relationships of flavonoids and phenolic acids. Free Radic Biol Med 20:933

Seyoum A, Asres K, El-Fiky FK (2006) Structure-radical scavenging activity relationships of flavonoids. Phytochemistry 67:2058

Sharma OP, Bhat TK (2009) DPPH antioxidant assay revisited. Food Chem 113:1202
Uma Devi P, Devipriya D, Murugan S, Selvi S, Suja S, Chinnaswamy P (2008) Evaluation of plasma total antioxidant response and total peroxides in different symptoms of schizophrenia patients. Int J Biol Chem 2:26

Wantusiak PM, Piszcz P, Skwarek M, Głód BK (2011) Antioxidative properties of honeys determined using HPLC techniques. Cam Sep 3:297 (in Polish)

Wantusiak PM, Piszcz P, Głód BK (2012) A fast and simple method for the measurement of total antioxidant potential and a fingerprint of antioxidants. J Chromatogr Sci 50:909

Zhang D, Chu L, Liu Y, Wang A, Ji B, Wu W, Zhou F, Wei Y, Cheng Q, Cai S, Xie L, Jia G (2011) Analysis of the antioxidant capacities of flavonoids under different spectrophotometric assays using cyclic voltammetry and density functional theory. J Agric Food Chem 59: 10277 